\title{
DEVELOPMENT OF A COHERENT BISTATIC VEGETATION MODEL FOR SIGNAL OF OPPORTUNITY APPLICATIONS AT VHF/UHF-BANDS
}

\author{
Mehmet Kurum (1), Manohar Deshpande ${ }^{(2)}$, Alicia T. Joseph ${ }^{(3)}$, \\ Peggy E. O'Neill ${ }^{(3)}$, Roger H. Lang ${ }^{(4)}$, Orhan Eroglu ${ }^{(1)}$ \\ (1) Department of Electrical and Computer Engineering, Mississippi State University, \\ Mississippi State, MS, 39762 \\ (2) Microwave Instrument Technology Branch (Code 555), NASA Goddard Space Flight Center, \\ Greenbelt, MD 20771 \\ (3) Hydrological Sciences Laboratory (Code 617), NASA Goddard Space Flight Center, \\ Greenbelt, MD 20771 \\ (4) Department of Electrical and Computer Engineering, George Washington University, \\ Washington, DC, 20052
}

\begin{abstract}
A coherent bistatic vegetation scattering model, based on a Monte Carlo simulation, is being developed to simulate polarimetric bi-static reflectometry at VHF/UHF-bands (240-270 MHz). The model is aimed to assess the value of geostationary satellite signals of opportunity to enable estimation of the Earth's biomass and root-zone soil moisture. An expression for bistatic scattering from a vegetation canopy is derived for the practical case of a ground-based/low altitude platforms with passive receivers overlooking vegetation. Using analytical wave theory in conjunction with distorted Born approximation (DBA), the transmit and receive antennas effects (i.e., polarization, orientation, height, etc.) are explicitly accounted for. Both the coherency nature of the model (joint phase and amplitude information) and the explicit account of system parameters (antenna, altitude, polarization, etc) enable one to perform various beamforming techniques to evaluate realistic deployment configurations. In this paper, several test scenarios will be presented and the results will be evaluated for feasibility for future biomass and root-zone soil moisture application using geostationary communication satellite signals of opportunity at low frequencies.
\end{abstract}

Index Terms - Bistatic, UHF, root-zone soil moisture, biomass, signal of opportunity, discrete scatterer.

\section{INTRODUCTION}

In the past decade, there has been increasing interest in using signals of opportunity (SoOp) for remote sensing of Earth [1]. Most of these past studies have focused on collection or modeling of Global Navigation Satellite Systems (GNSS) signal reflections over ocean surface for estimating wind direction and speed or altimetry. On the other hand, application of GNSS-Reflectometry technique to land is rather scarce and relies mostly on experimental observations [2-4]. Sensitivity to soil and vegetation by GNSS's bistatic signals at L-band has been, to some extent, experimentally demonstrated and have been also supported by few theoretical studies [5-9]. However, L-band signals are limited to penetrate through dense vegetation and deep into the soil column. Thereby, GNSS-based approaches can provide estimate of surface soil moisture up to a depth of $\sim 0.05$ meter through moderate amounts of vegetation. In recent past, to address this issue, a new SoOp methodology is suggested, where the signals transmitted by the Military Satellite Communication (MilSatCom) System's UHF (240$270 \mathrm{MHz}$ ) Follow-On program) are utilized to measure properties of reflecting targets by recording reflected signals using a simple passive microwave receiver $[10,11]$.

The low frequency SoOP approach has the potential of measuring root-zone soil moisture (top 1-m) through heavy vegetation. These measured quantities would provide information critical for understanding global water resources and the vertical moisture gradient responsible for evapotranspiration and runoff. In this study, a low frequency (VHF/UHF) bi-static electromagnetic scattering model is being developed to supplement recent experimental efforts and to exploit further their potential. Since the low-frequency SoOP experiments are so far carried out from either ground based or airborne platforms, we will restrict the modeling study to such geometries, but it can be fairly easily extended to the receivers in low Earth orbit. The model will be used to conduct sensitivity analysis and to determine optimum system configuration for 
estimating root-zone soil moisture and biomass parameters for the Earth surface covered with heavy forest/vegetation.

The antenna characteristics and orientation play a key role in received signal. For ground-based systems, the antenna radiation pattern projected on the surface is not uniformly distributed in phase, amplitude, or polarization. The bistatic radar coefficient calculated by the model thus needs to have the antenna characteristics embedded in it as well as the statistical and physical properties of the terrain to mimic the real measurement setting. Previous studies have usually assumed plane wave illumination/scattering [5-7], and ignored such antenna effects. In the following section, a brief description of the model will be provided for calculating the response of the antenna due to the coherent bistatic scattered field from a vegetated surface.

\section{FORMULATION}

In this paper, a general bistatic scattering case is considered, where two antennas do not have their main beam axis pointing at each other and both of which are overlooking the vegetation. One antenna system is associated with the signal of opportunity transmitter while the other refers to the passive receiver. The vegetation is represented as ensemble of canonical scatterers located above a flat ground. The transmitter is located far away. The local incident angles are assumed to be constant (parallel) and spreading loss effects due to the incoming wave are ignored, but the spreading loss and sphericity of the scattered wave are considered due to proximity of the platforms that operate close to the ground. This configuration will represent "plane wave incidence" and "spherical wave scattering".

It is important to realize that antennas cannot be constructed to produce pure polarization states. There will always be some nonzero cross polarization level that could degrade the measurement. Additionally, the polarization state of the antenna is usually defined along the main beam direction. The polarization, however, varies for increased off-axis angles over the radiation pattern. Polarization states of the antennas are taken into account through a polarization (field) rotation matrices. As a result, the formulation considers variations of both the strength and polarization states of the received wave along the beam direction.

Applying 'discrete scatter' modeling approach [12], the forest layer is replaced by a slab of equivalent homogenous medium by the Foldy theory [13]. An expression for the scattered field due to a single particle immersed in the slab of mean medium over a flat ground surface is derived. The received signal is then expressed as a weighted coherent sum involving the antenna spatial spectrum and bistatic scattering. The results of the single particle scattering are summed over all types and particles by taking into account of the polarization mixing (rotation matrices), the antenna

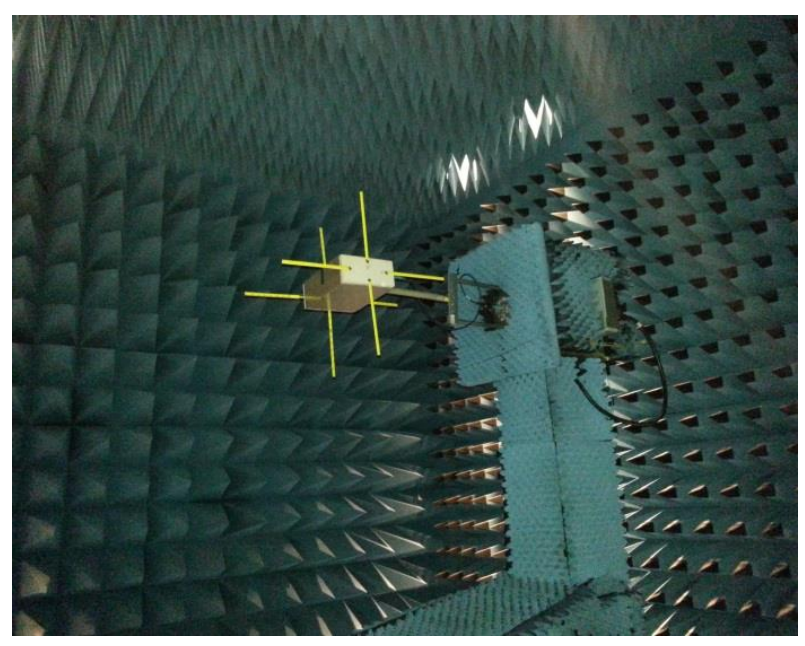

Figure 1: 4-channel VHF receiver.

impurities (antenna pattern matrices with co- and cross-pol components), and the spreading loss.

The received field has three main terms: (1) direct term, (2) specular term, (3) diffuse term. The first one represents the line of sight or the shortest path between the antennas while the second term denotes the forward scattering along the specular direction, where the incoming and the scattering waves make the same angle with respect to the surface normal. Lastly, the diffusely scattered waves arrive at the receiver antenna from a wide range of angles in both azimuth and elevation due to scattering from the illuminated volume. An average diffuse term is obtained by a sufficient number of realizations of vegetation through Monte Carlo simulations.

Each term is a "complex" field quantity and thus includes both amplitude and phase information. This enables one to decompose the field into a orthogonal polarization basis in order to simulate polarimetric bi-static reflectometry for various polarization combinations.

\section{SIMULATION SETTINGS}

In the simulation, several scenarios will be tested for the recently developed 4-channel VHF receiver hardware [11]. The instrument is shown in anechoic chamber for measurement of radiation characteristics in Fig. 1. Each channel receives VHF signal picked up by the linear dipole tuned to frequency range 240-270 MHz. Due to broad beam dipole patterns, each channel will receive the direct as well as reflected signal (reflected from the vegetation) transmitted by the MilSatCom satellites. The instrument is capable of recording complex field quantities that allows to apply beamforming. Using digital beam forming in the post processing, the direct signal and the reflected signals are separated for soil moisture and root zone soil moisture extraction. 
Zenith, direct signal, horizon, and reflected signal directions will be considered for antenna orientations. For each configuration, the 4 channel array beam will be steered into direct and reflected direction to isolate the signals. The model will be simulated for considering various combination of system and target parameters. Among the antenna parameters, effects of antenna height, antenna cross-polarization, and beamwidth width will be analyzed to understand platform and antenna requirements for such applications. For each system configuration, the target parameters such as soil moisture, vegetation type, height, and biomass level, will be varied to conduct sensitivity analysis.

\section{CONCLUSION}

Knowledge of the Earth biomass, root zone soil moisture (including surface soil moisture) are important earth science parameters because of their strong influence on the Earth's climate system. Currently, these Earth science parameters are measured using airborne and space borne microwave radiometers/scatterometers operating at L-band $(1.4 \mathrm{GHz})$ frequencies. However, the L-band frequency range is inadequate for measuring these parameters for the Earth surface covered with heavy forest/vegetation. In the recent past, NASA has shown great interest in using lower frequency band such as VHF/UHF for these parameter measurements. In this study, newly developed coherent bistatic vegetation scattering model, based on a Monte Carlo simulation, will be utilized to simulate polarimetric bi-static reflectometry at VHF/UHF-band. Preliminary performance of the model and sensitivity analysis by the model will be presented.

\section{REFERENCES}

[1] U. Zavorotny, S. Gleason, E. Cardellach, and A. Camps, "Tutorial onremote sensing using GNSS bistatic radar of opportunity," IEEE Geosci. Remote Sens. Mag., vol. 2, no. 4, pp. 8-45, Dec. 2014.

[2] A. Alonso-Arroyo, A., Camps, A. Aguasca, G.F. Forte, A. Monerris, C. Rudiger, J.P. Walker, H. Park, D. Pascual, R. Onrubia, "Dual-polarization GNSS-R interference pattern technique for soil moisture mapping," IEEE J. Sel. Topics Appl. Earth Obs. Remote Sens., vol. 7, no. 5, pp. 1533-1544, May 2014.

[3] A. Egido, S. Paloscia, E. Motte, L. Guerriero, N. Pierdicca, M. Caparrini, E. Santi, G. Fontanelli, N. Floury, "Airborne GNSS-R polarimetric measurements for soil moisture and above-ground biomass estimation," IEEE J. Sel. Topics Appl. Earth Obs. Remote Sens., vol. 7, no. 5, pp. 1522-1532, May 2014.

[4] A. Camps, H. Park, M. Pablos, G. Foti, C. P. Gommenginger, P. W. Liu, and J. Judge, "Sensitivity of GNSS-R spaceborne observations to soil moisture and vegetation," IEEE J. Sel. Topics
Appl. Earth Observ. Remote Sens., vol. 9, no. 10, pp. 4730-4742, Oct. 2016.

[5] P. Liang, L.E. Pierce, and M. Moghaddam, "Radiative transfer model for microwave bistatic scattering from forest canopies," IEEE Trans. Geosci. Remote Sens., vol. 43: pp. 2470-2483, 2005.

[6] P. Ferrazzoli, L. Guerriero, N. Pierdicca, and R. Rahmoune, "Forest biomass monitoring with GNSS-R: Theoretical simulations," Adv. Space Res., vol. 47, no. 10, pp. 1823-1832, 2010.

[7] L. Guerriero, N. Pierdicca, L. Pulvirenti, and P. Ferrazzoli, "Use of satellite radar bistatic measurements for crop monitoring: A simulation study on corn field," Remote Sens., vol. 5, pp. 864890,2013

[8] N. Pierdicca, L. Guerriero, R. Giusto, M. Brogioni, and A. Egido, "SAVERS: A simulator of GNSS reflections from bare and vegetated soils," IEEE Trans. Geosci. Remote Sens., vol. 52, no. 10, pp. 6542-6554, Oct. 2014.

[9] X. R. Wu and S. G. Jin, "GNSS-Reflectometry: Forest canopies polarization scattering properties and modeling," Adv. Space Res., 54(5), 863-870, 2014.

[10] J. Knuble, J. Piepmeier, M. Deshpande, C. D. Toit, J. Garrison, Y-C. Lin, G. Stienne, S. Katzberg, G. Alikakos, "Airborne P-band Signal of Opportunity (SoOP) demonstrator instrument; status update," in Proc. IEEE Int. Geoscience Remote Sensing Symp., Beijing, China, 2016.

[11] A. T. Joseph, M. Deshpande, P. E. O’Neill, and L. Miles, "Development of VHF (240-270 MHz) Antennas for SoOp (Signal of Opportunity) Receiver for 6U Cubesat Platforms," in Proc. Progress In Electromagnetic Research Symposium (PIERS), Shanghai, China, 2016.

[12] M. Kurum, (2009). L-band Estimation of Forest Canopy Attenuation by a Time-Domain Analysis of Radar Backscatter Response. Chapter 3, Ph.D. dissertation. The George Washington University. Washington DC, US.

[13] L. Tsang, J. Kong, and R. Shin, Theory of Microwave Remote Sensing. ch. 3, New York, Wiley, 1985. 